\title{
Alcances de la Enseñanza de la Estadística a través de la Investigación en la Educación Media en Venezuela
}

\author{
Nelly León Gómez \\ nellyleong@hotmail.com \\ https://orcid.org/0000-0001-8500-1253 \\ Universidad Pedagógica Experimental Libertador \\ Instituto Pedagógico de Maturín "Antonio Lira Alcalá" \\ Maturín, Venezuela
}

Recibido: 07/05/2020 Aceptado: 07/05/2020

\begin{abstract}
Resumen
El tema central de este artículo tiene que ver con la pertinencia de una enseñanza de la Estadística a través de la investigación con miras a la formación de un ciudadano crítico. Primeramente se hace una revisión de los elementos conceptuales que permiten posicionar a la Estadística como la ciencia de los datos y la incertidumbre, por lo que hacer estadística implica crear significados a través de datos en contexto, poniendo en juego el pensamiento y el razonamiento estadísticos. Luego se pasa a cuestionar la forma como se enseña Estadística en Venezuela en Educación Media, apegada a la tradición calculista y procedimental. A través de un caso particular se busca poner en evidencia las limitaciones y las oportunidades de los docentes para una enseñanza/aprendizaje de la disciplina con la aplicación de la investigación estadística a través de proyectos de aprendizaje. Para cerrar se destaca la necesidad de introducir cambios en la formación del docente de Matemática que conlleven a adoptar un modelo que contemple por una parte el conocimiento conceptual y pedagógico para la enseñanza y por otra el desarrollo del pensamiento estadístico de los propios docentes, sin olvidar el componente afectivo de la Educación
\end{abstract}

Palabras clave: Investigación Estadística. Alfabetización, Razonamiento y Pensamiento Estadístico. Enseñanza por Proyectos. Formación Docente.

\section{Escopo do Ensino de Estatística através de Pesquisas no Ensino Médio na Venezuela}

\section{Resumo}

O tema central deste artigo tem a ver com a relevância de um ensino de estatística por meio de pesquisas. Primeiramente, é feita uma revisão dos elementos conceituais que permitem que a Estatística seja posicionada como a ciência dos dados e da incerteza, de modo que a estatística implica a criação de significados através dos dados no contexto, colocando o pensamento e o raciocínio estatísticos em jogo. Em seguida, questionamos o modo como as estatísticas são ensinadas na Venezuela no ensino médio, ligadas ao cálculo e à tradição processual. Através de um caso particular, o objetivo é destacar as limitações e oportunidades dos professores para o ensino / aprendizagem da disciplina com a aplicação da pesquisa estatística por meio de projetos de aprendizagem. Para encerrar, destaca-se a necessidade de introduzir mudanças na formação dos professores de Matemática que levem à adoção de um modelo que contemple, por um lado, o conhecimento conceitual e pedagógico do ensino e, por outro, o desenvolvimento do pensamento estatístico dos próprios professores.

Palavras chave: Pesquisa Estatística. Alfabetização, Raciocínio e Pensamento Estatísticos. Ensino por Projeto. Formação de Professores. 


\title{
Scope of Teaching Statistics through Inquiry in Middle School of Venezuela
}

\begin{abstract}
This article deal with the relevance of teaching Statistics through investigation. Firstly, we make a review of the conceptual framework that allows us to define Statistics as the science of data and uncertainty, so making statistics implies the creation of meanings through data in context, highlighted by the statistical thinking and reasoning. Then, we go on to question the way Statistics is taught in Venezuela in middle school, as being a part of the mathematics curriculum. Through a particular case, we highlight the limitations and opportunities of teachers for teaching the statistical matter through inquiry in the context of learning projects. Finally, we propose changes in the preparation of Mathematics teachers that lead to adopting a model that comprises on the one hand the conceptual and pedagogical knowledge for teaching Statistics, and on the other the development of their own statistical thinking.

Keywords: Statistical Investigation. Statistical Literacy, Reasoning, and Thinking. Learning Projects. Teachers Training.
\end{abstract}

\section{Para comenzar, una digresión: conviviendo con la incertidumbre}

Escribo este artículo en plena crisis del Coronavirus, guardando la cuarentena social comunitaria decretada por el gobierno venezolano como medida para controlar la propagación de este poderoso virus en el país. Confieso que no ha sido fácil concentrarme en esta tarea a pesar del tiempo disponible. Hay muchos elementos emocionales unidos a la sensación de incertidumbre que me llevan a ocupar estos momentos de reclusión en pensamientos y reflexiones sobre lo inédito de la situación actual y los cambios y transformaciones que se suscitarán a nivel planetario en los ámbitos social, político, económico y medioambiental.

Siempre he estado consciente de que constantemente transitamos por circunstancias inciertas, pero con esta pandemia la incertidumbre nos ha dado una bofetada que ha puesto a tambalear cualquier resquicio de certeza que pudiera quedar en relación a nuestra forma de vivir y convivir en este mundo globalizado (¡Cómo se ha aprovechado el Covid-19 de esta globalización!) y nos abre la puerta a una aventura (quizás terrorífica), hasta ahora desconocida para nosotros en los tiempos que nos ha tocado vivir.

Sigo a Edgar Morin en las redes sociales, y durante este período de reclusión impuesto, o autoimpuesto, he compartido algunos de sus pensamientos y reflexiones. En un tweet publicado el 20-03-2020, Morin escribió sobre las grandes lecciones de esta crisis: "No poder escapar de la incertidumbre; todavía estamos en la incertidumbre de la cura del virus; en la 
incertidumbre de los desarrollos y las consecuencias de la crisis. Permaneceremos en la incertidumbre de la condición humana". (@edgarmorinparis)

La crisis actual ha superado cualquier expectativa, llevando nuestra incertidumbre a una máxima expresión. También dice Morin (@edgarmorinparis, 20-03-2020) “El universo rompe sobre nosotros cruzando los muros de nuestro confinamiento", y en este confinamiento permanecemos atados a la televisión y a las redes sociales como posibilidades para cruzar esos muros y conocer lo que ocurre más allá de las cuatro paredes que nos albergan. Pero, ¿qué ocurre realmente allá afuera? Son tan diversas y a veces tan contradictorias las noticias que circulan que contribuyen más a desinformar que a informar; el manejo de las cifras asociadas a la enfermedad producida por el Covid-19 genera un ambiente de zozobra en la población, ya de por si emocionalmente afectada, y potencia la incertidumbre reinante sobre nuestro porvenir individual y colectivo. Surgen tantas interrogantes y tal diversidad de respuestas a cada una de ellas, "respaldadas en datos", que quizás el ciudadano común no pueda procesarlas convenientemente.

Siempre se ha dicho que la información es poder, pues permite la toma de decisiones y la actuación acertada en condiciones de incertidumbre, pero cuando no se tiene confianza en los datos que se reportan sobre un acontecimiento, entonces se produce un efecto contrario que puede conducir a decisiones inapropiadas e inoportunas si no se está preparado para el manejo y comprensión de dicha información. Es indispensable, entonces, que todo ciudadano tenga una cultura estadística que le brinde las herramientas para pensar por sí mismo, hacer preguntas inteligentes y confrontar a la autoridad con confianza (Steen, 2001; citado por Franklin, Kader, Mewborn, Moreno, Peck, Perry y Scheaffer (2005). En las circunstancias actuales se requeriría una postura crítica sobre los datos, su procedencia, el análisis que de ellos se ha hecho y los resultados que se publican.

Por ejemplo, en Venezuela, golpeada desde hace ya bastante tiempo por una grave crisis social, política y económica, toda persona con una mínima cultura estadística debe mostrar cierto escepticismo ante las cifras oficiales sobre la pandemia en nuestro país: ¿Podemos aceptar sin reservas la información del ejecutivo nacional en cuanto al número de personas contagiadas, recuperadas y fallecidas, la cantidad de pruebas realizadas para detectar casos positivos y las condiciones de nuestro sistema de salud para la atención de los pacientes con el nuevo virus (número de camas hospitalarias disponibles, respiradores, tratamiento, personal de salud, etc.)? 
¿Cómo se comparan estos datos con las experiencias de otros países que están sufriendo seriamente los embates de esta crisis?

Las respuestas a estas inquietudes no deben darse desde posturas políticas sino a partir de una revisión crítica de los datos, con las herramientas de pensamiento y razonamiento que nos provee la Estadística como ciencia de aprender de los datos y de medir, controlar y comunicar la incertidumbre, como la concibe la American Statistical Asociation (ASA), de allí la importancia de la Estadística en la formación crítica de los ciudadanos.

\section{Entrando en materia: la Estadística en la formación de ciudadanos críticos}

En Noviembre de 2019, la UNESCO aprobó decretar el 14 de marzo (3/14) como el Día Internacional de las Matemáticas / International Day of Mathematics (IDM) con el fin de destacar el importante papel que éstas desempeñan en la comprensión de los principales desafíos sociales y ambientales del mundo en la actualidad (UNESCO). El tema central para la conmemoración de esta fecha en 2020 ha sido "Mathematics are everywhere" o "las matemáticas están en todas partes", como una forma de visualizar la presencia de las matemáticas en la cotidianidad.

En la sociedad actual, lo mismo podemos decir de la estadística, "la Estadística está en todas partes" pues ésta arropa cada una de las facetas de la vida humana. Transitamos por la era de la información y la comunicación, y ambas se sustentan en los datos que se generan en los más variados contextos y situaciones, sean estos políticos, sociales, económicos, laborales, científicos.

La Estadística es reconocida como la ciencia de los datos (Moore, Mc Cabe y Craig, 2009), como un método fundamental de investigación y como una forma específica de pensar (Moore y Cobb, 1997). El propósito de la Estadística en la práctica es ganar comprensión de situaciones contextuales a partir de datos generados de tales situaciones (Moore, et al, 2009; Ben-Zvi y Makar, 2016), lo que conllevaría a decisiones razonadas en entornos de incertidumbre.

Para que el conocimiento de información relevante se convierta en un arma a favor del que la posee, se debe contar con conocimientos y herramientas cognitivas que permitan extraer sentido de los datos, separando lo relevante de lo superfluo, cuestionar su procedencia, comprender las formas en que son representados y procesados y las interpretaciones de los significados que se les asignan y de las conclusiones y explicaciones de las cuestiones de interés. 
La estadística abre las posibilidades de desarrollar formas de razonar partiendo de datos empíricos inciertos y formas de pensar en términos de la variabilidad de los fenómenos y de patrones que en ellos emergen, lo que le facilitará moverse en condiciones de incertidumbre, por lo que debería formar parte del bagaje cultural de todo ciudadano educado.

Son muchos los investigadores que destacan la importancia que tiene para toda persona contar con una cultura estadística derivada de los conocimientos y las formas de pensar y razonar que le permitan comprender el mundo en que vive (Batanero, 2004), y ejercer a plenitud su ciudadanía (Franklin et al, 2005; Gal, 2002).

Ya para finales del siglo XIX, Wells, citado por Watson (1997, p. 107) predecía que "El pensamiento estadístico algún día será tan necesario para una ciudadanía eficiente como la capacidad de leer y escribir ", En 1993, Katherine Wallman introduce el término alfabetización estadística para referirse a

La habilidad para comprender y para evaluar de forma crítica los resultados estadísticos que permean nuestra vida diaria, junto con la habilidad para apreciar la contribución que el pensamiento estadístico puede aportar tanto a nivel público como privado en la toma de decisiones profesionales y personales. (Wallman, 1993, p. 1)

Por su parte, Watson (1997) resalta como componentes del pensamiento estadístico: el conocimiento de los conceptos estadísticos, la comprensión de las formas de razonar estadísticamente y una actitud crítica para cuestionar argumentos, resultados y conclusiones basados en evidencia estadística no suficiente.

La Educación Estadística en la actualidad está llamada, precisamente, a equipar a los estudiantes de marcos conceptuales, a promover formas de pensar y razonar propios de la Estadística y a desarrollar habilidades de la práctica estadística para navegar exitosamente en las turbulentas aguas de la sociedad en que vivimos (Wild, Utts y Horton, 2018; Estrella, 2017).

El reto de los educadores es grande pues implica cambiar de un esquema de enseñanza centrado en cálculos y aplicación de fórmulas a otro basado en datos. Esto amerita no solo cambios curriculares, sino también en la formación de los docentes y en las actitudes y concepciones tanto de profesores como de los estudiantes hacia la disciplina y su enseñanza y aprendizaje. Es notorio que la estadística es vista por los estudiantes como algo difícil de aprender y sin notables aplicaciones en su actuar diario, y por los docentes como una materia 
difícil de enseñar y no imprescindible en la formación de los niños y jóvenes (Ben-Zevi y Garfield, 2004).

Posiblemente, mucho de este rechazo se debe al hecho de que los contenidos de esta área generalmente están incluidos en el currículo de Matemática y en consecuencia son enseñados por profesores de Matemática que siguen la tradicional metodología de esta disciplina, poniendo énfasis en cálculos y fórmulas que los estudiantes usualmente no dominan y en la búsqueda de respuestas correctas a los ejercicios descontextualizados que plantea el docente (Ben-Zevi y Garfield, 2004; Sánchez y Hoyos, 2013). Sin embargo, muchos educadores estadísticos coinciden en señalar que la Estadística no es una rama de la Matemática aun cuando sea una disciplina matemática que se nutre de ella, pero que se diferencia en cuestiones esenciales como el rol del contexto, formas de razonamiento, los datos, su rol y su generación y la precisión, entre otras (Batanero, 2000; Burril y Biehler, 2011; DelMas, 2004; Franklin et al, 2005; Gattuso y Ottaviani, 2008 y Scheaffer, 2006).

El enfoque calculista de ninguna manera permite captar la Estadística en su verdadera dimensión como la ciencia de los datos y de la incertidumbre; por el contrario, hace llegar a los estudiantes una Estadística carente de sentido. En los tiempos actuales la enseñanza de la estadística se deberá realizar en entornos de aprendizaje activo que involucren a los estudiantes en estudios estadísticos, en comprender cómo conducirlos y cómo interpretar sus resultados (Azcárate y Cardeñoso, 2011; Utts, 2003) En tal sentido, se debe apuntar a la enseñanza y comprensión de las ideas estadísticas fundamentales: datos, variación, distribución, representación, asociación y modelaje de relación entre variables y la probabilidad para el estudio de modelos en el proceso de generación de datos. (Burril y Biehler ,2011; Da Ponte y Noll, 2018; Shaughnessy, 2019)

El conocimiento estadístico es un conocimiento contexto-dependiente, lo que quiere decir que su interpretación depende del contexto de aplicación (Azcárate y Cardeñoso, 2011). En la actualidad, las recomendaciones en torno a la enseñanza de la Estadística, insisten en la conveniencia de alejar esta disciplina de una enseñanza matematizada para pasar a privilegiar la interpretación y comprensión que conllevan al desarrollo del razonamiento y el pensamiento estadísticos (Garfield y Ben Zevi, 2008) y esto se puede lograr apelando a la contextualización, la participación activa de los estudiantes y el trabajo cooperativo, todo esto con el auxilio de la tecnología. 


\section{Una trilogía fundamental de la Educación Estadística: Alfabetización, razonamiento y pensamiento estadísticos.}

La literatura sobre estas tres manifestaciones de una cultura estadística es tan prolífera como ambigua. Su revisión nos lleva a percatarnos de que no existe claridad respecto a la definición de cada uno de estos términos y de sus alcances, los cuales aparecen muchas veces sobrelapados al asignar las mismas capacidades al razonamiento y al pensamiento estadístico o asociar la alfabetización estadística con la alfabetización numérica (Ben Zevi y Garfield, 2004); más aún, algunos los usan indistintamente en un intento de diferenciar la comprensión de los conceptos estadísticos de la manipulación numérica que usualmente caracteriza la enseñanza de la disciplina (Chance, 2002).

Para autores como Garfield (2002), del Mass (2002) y Chance (2002), la alfabetización estadística se traduce en la capacidad de leer, interpretar y evaluar información de los periódicos y de la vida diaria con el uso del lenguaje estadístico básico y las representaciones en tablas y gráficos. También incluye la comprensión de símbolos, vocabulario, conceptos estadísticos y la probabilidad como una medida de incertidumbre (Ben-Zevi y Garfield, 2004)

El razonamiento estadístico lo vamos a entender como el tipo de razonamiento asociado a los diversos conceptos estadísticos y a ser capaz de conectar diferentes ideas y conceptos estadísticos y comprender y explicar los procesos estadísticos como por ejemplo el de la producción de los datos (Garfield y Ben-Zevi, 2008). Implica ser capaz de explicar porqué un resultado específico es esperado o ha ocurrido; porqué es apropiado seleccionar un modelo particular y si este se ajusta razonablemente al contexto en estudio (delMas, 2004).

El pensamiento estadístico viene a ser el tipo de pensamiento que se pone en juego ante un problema estadístico y abarca: considerar la naturaleza de los datos, su procedencia y su fiabilidad; seleccionar las técnicas y modelos de análisis estadístico más apropiados a los datos; interpretar los resultados de dichos análisis en el contexto del problema; y comunicar los resultados de dicho análisis con el uso del lenguaje estadístico. Implica la comprensión de cómo y porqué se realiza la investigación estadística, la consideración de la omnipresencia de la variación en todos los fenómenos, cómo y cuándo usar los métodos estadísticos, entre otras habilidades que capacitan para evaluar críticamente resultados de estudios estadísticos (Garfield y Ben-Zevi, 2008). 
Chris Wild y Maxime Pfannkuch (Wild y Pfannkuch, 1999; Pfannkuch y Wild, 2004) presentan un marco para el pensamiento estadístico consistente en cuatro dimensiones:

Dimensión 1: Ciclo investigativo (PPDAC), que comprende Problema (P): planteamiento del problema y pregunta de investigación; Plan (P): planificación del diseño, recolección de datos y análisis; Datos (D): recolección, manejo y limpieza de los datos; Análisis (A): exploración de datos, análisis de los datos, enunciado y contraste de hipótesis; Conclusión (C): Interpretación, conclusión, nuevas ideas, comunicación.

Dimensión 2: Tipos fundamentales de pensamiento estadístico: 1) Reconocimiento de la necesidad de los datos: 2) Transnumeración, o comprensión que surge de cambiar la representación de los datos; 3) Percepción de la variación, que implica la búsqueda de patrones en la variación y su comprensión en el marco del contexto donde se origina; 4) Razonamiento con modelos, en tanto que estos permiten explorar y detectar patrones en los datos y resumir datos en muy variadas formas; y 5) Integración de la Estadística y el contexto, como una componente esencial del pensamiento estadístico.

Dimensión 3: Ciclo interrogativo, que comprende: Generar, buscar, interpretar, criticar y juzgar Dimensión 4: Disposiciones, asociadas a las formas como los estudiantes abordan un problema estadístico: Escepticismo, imaginación, curiosidad y conciencia, apertura, propensión a buscar un significado más profundo, ser lógico, compromiso y perseverancia.

En los tiempos actuales, la educación estadística debe estar orientada hacia la formación de un ciudadano estadísticamente alfabetizado y con niveles de razonamiento y pensamiento estadísticos que lo capaciten para convivir exitosamente en ambientes de incertidumbre.

\section{Proyectos e investigación estadística: o cómo desarrollar el pensamiento estadístico}

Como ya hemos puntualizado, el conocimiento estadístico cobra su sentido pleno mediante su aplicación a situaciones contextualizadas, cotidianas y en su relación con otras disciplinas. Según lo establecido en el proyecto GAISE (Franklin et al, 2005), en la actualidad prevalece la tendencia internacional de ubicar ese conocimiento conceptual en un contexto académico basado en datos, mediante un aprendizaje activo y con el uso de la tecnología, a través de la de resolución de problemas en los cuales los estudiantes se involucren en cada una de las etapas de una investigación estadística (PPDAC): formulación de preguntas de investigación; diseño del estudio; recolección, organización y representación de datos; selección 
y aplicación de las técnicas estadística apropiadas; análisis de los resultados y comunicación de conclusiones y predicciones basadas en los datos. (Insunza, 2014)

La Estadística provee herramientas para la resolución de problemas reales, el tratamiento de la información, la toma de decisiones, el desarrollo de la capacidad de comunicación y el trabajo en equipos, todas éstas, habilidades que se fomentan mediante el desarrollo de proyectos o investigaciones estadísticas (Batanero y Godino, 2005).

La enseñanza por proyectos comulga con el principio de promover entornos de aprendizaje activo y colaborativo, en los cuales los estudiantes se involucran en actividades de búsqueda, reflexión, cuestionamiento y discusión (NCTM, 2000; Azcárate y Cardeñoso, 2011; Ponte, 2011).

Con los proyectos, la Estadística cobra sentido más allá del aula, mediante su aplicación a situaciones de interés del conglomerado estudiantil. De esta manera, se aleja a los estudiantes de la creencia de que la Estadística se reduce a números carentes de sentido sobre los cuales se aplican cálculos rutinarios y tediosos (Díaz y Sánchez, 2011; Díaz, Aguayo y Cortez, 2014), para acercarlos a una nueva visión como "una forma de razonar (el razonamiento que en situaciones de incertidumbre permite realizar inferencias y guiar la toma decisiones a partir de los datos)" (Batanero, 2002, p. 1).

Holmes (1997, citado por Batanero, Díaz, 2011), entre los aportes de esta estrategia de enseñanza y aprendizaje, destaca las posibilidades de contextualización, de despertar el interés de los estudiantes y de promover el razonamiento estadístico al trabajar con datos que surgen de un problema real, no impuesto por el profesor, que el estudiante quiere resolver y que a su vez permite introducir ideas estadísticas como precisión, variabilidad, fiabilidad, posibilidad de medición, sesgo, entre otras, que no surgen cuando se usan datos inventados por el profesor sobre los cuales se realizan cálculos matemáticos.

La enseñanza de la estadística mediante proyectos propicia el desarrollo del pensamiento estadístico a través del proceso de investigación estadística (Batanero y Díaz, 2011; MacGillivray y Pereira-Mendoza, 2011). Precisamente el trabajo con proyectos estadísticos comienza con el planteamiento de una situación-problema, siendo preferible que esta emerja de los mismos estudiantes de acuerdo a sus intereses comunes; esto llevará al planteamiento de la pregunta o preguntas iniciales de investigación. Huelga decir que ésta es una de las etapas más difíciles del proceso, sobre todo tomando en cuenta que los estudiantes están más acostumbrados a seguir 
instrucciones que a reflexionar sobre los alcances de las tareas que tienen que asumir. El tipo de preguntas orientará las etapas siguientes del proceso: recogida de información; organización, análisis e interpretación de los datos recopilados (según el contexto del proyecto y evitando el trabajo manual innecesario); responder las preguntas iniciales y comunicar resultados y conclusiones.

Esto requiere concordancia entre la concepción y la ejecución de acuerdo a las particularidades de la situación que se aborda, moverse bidireccionalmente entre la teoría y la práctica, y manejar la complejidad y la incertidumbre (Ponte, 2011). El papel del docente es el de guía y orientador; aprovechará cada ocasión para, después de la discusión en el contexto del proyecto, institucionalizar el conocimiento estadístico que se vaya requiriendo y a su vez promover el desarrollo del razonamiento y el pensamiento estadístico.

\section{¿Posibilidad o utopía?: Enseñanza de la Estadística a través de proyectos en Venezuela}

A partir de la reforma del nivel de Educación Media en Venezuela, que llevó a la implantación de un nuevo diseño curricular, desde el año 2014 se ha buscado cambiar la visión negativa que prevalecía sobre la enseñanza de la Matemática al tratar de mostrar su utilidad en la comprensión de los fenómenos naturales y sociales (MPPE, 2015). En el currículum de este nivel, por una parte, se concibe la Matemática como "una disciplina humana y, culturalmente hablando, útil en todos los sentidos, indispensable y necesaria para la vida particular de las personas, para la colectividad y para la sociedad” (MPPE, 2015, p. 213). Es decir, se busca visualizar que "las matemáticas están en todas partes", y por otro, siguiendo a Skovsmose (1999) se plantea una Educación Matemática que permita a los ciudadanos ser parte de una sociedad democrática, actuando en ella de una manera crítica y comprometida con las transformaciones necesarias para garantizar el bienestar colectivo conjuntamente con la preservación del ambiente.

En este escenario se comulga tanto con la filosofía de la Educación Matemática Crítica como con los principios de la Educación Matemática Realista: es decir, se aboga por un realismo crítico en la Educación Matemática que "establezca una estrecha relación entre los fenómenos sociales o naturales y la enseñanza de las matemáticas vista desde diferentes perspectivas, tomando en cuenta .... la conectividad interna de las matemáticas y la incorporación del mundo extramatemático" (Mora, 2005, citado en MPPE, 2015, p. 244). 
El contenido curricular se engloba en cuatro organizadores: forma y dimensión, cambio, cantidad e incertidumbre. Este último organizador, incertidumbre, se considera indispensable para la formación de un ciudadano crítico; comprende la Estadística y la Probabilidad como las disciplinas de los datos y el azar y por lo tanto incluye los conceptos y procedimientos para el manejo de datos: recolección, representación, análisis; la probabilidad y la inferencia. (MPPE, 2015).

La enseñanza de la Matemática en general, y en particular la Probabilidad y la Estadística, debe promover el desarrollo de capacidades para la indagación, para la interpretación, la toma de decisiones y para discernir sobre el uso apropiado o no de los saberes de esta disciplina. Con el organizador Incertidumbre se espera que los estudiantes sean capaces de "Resolver problemas que permitan procesar información partiendo de su cotidianidad, formular hipótesis, crear patrones, modelos, hacer representaciones y llegar a conclusiones como parte de construcciones colectivas" (MPPE, 2015, p. 261).

El currículum de Educación Media en Venezuela se organiza atendiendo entre otros a los siguientes elementos: temas generadores, tejido temático y referentes teórico-prácticos. Los temas generadores se refieren a cuestiones macro que propician aprendizajes integrados y contextualizados. El tejido temático está constituido por un mapa de posibles aspectos interdisciplinares factibles de ser considerados en el abordaje de los temas disciplinares de cada área. Los referentes teórico-prácticos engloban los conocimientos propios del área (MPPE, 2015). Los temas generadores propician aprendizajes significativos de los referentes teóricos prácticos enlazados a los considerados temas indispensables a través del tejido temático; con lo que se busca la integración interdisciplinar a través de la Matemática. En este contexto se debe privilegiar la enseñanza por proyectos, donde la estadística debería jugar un papel importante.

En el Cuadro 1 se presenta la organización curricular de Matemática, correspondiente a las unidades académicas 1 y 5 de primer año de Educación Media, que incluyen los referentes teórico-prácticos del organizador Incertidumbre. 
Cuadro 1: Unidades curriculares del programa de $1^{\circ}$ año de Educación Media vinculadas al organizador Incertidumbre.

\begin{tabular}{|c|c|c|}
\hline UA & Tema generador-Tejido temático & Referentes teórico-prácticos \\
\hline 1 & $\begin{array}{l}\text { La Estadística y sus aplicaciones } \\
\text { Aplicación de la estadística en las diversas áreas } \\
\text { Descripción, organización y visualización de datos originados a } \\
\text { partir de la indagación. } \\
\text { Generación de modelos, inferencias y predicciones asociadas a } \\
\text { los fenómenos indagados. } \\
\text { ¿Qué, para qué, cuándo, dónde y cómo recolectar datos? }\end{array}$ & $\begin{array}{l}\text { Estadística descriptiva e } \\
\text { inferencial } \\
\text { Instrumentos de recolección de } \\
\text { datos } \\
\text { Tablas de doble entrada } \\
\text { Representaciones gráficas de } \\
\text { proporciones } \\
\text { Estimaciones, generalizaciones } \\
\text { Porcentajes }\end{array}$ \\
\hline 5 & $\begin{array}{l}\text { Alimentación y nutrición } \\
\text { Influencia del programa de la alimentación escolar en el } \\
\text { crecimiento. } \\
\text { Forma para medir la alimentación necesaria. Tabla de calorías. } \\
\text { Trompo de los alimentos } \\
\text { Patrones de consumo. } \\
\text { Desigualdad al acceso del alimento en el mundo. } \\
\text { El índice de masa corporal y su relación con algunas } \\
\text { enfermedades. } \\
\text { Sustancias dañinas para la salud y composición. }\end{array}$ & $\begin{array}{l}\text { Tablas } \\
\text { Gráficas } \\
\text { Media aritmética } \\
\text { Razones, proporciones } \\
\text { Índices } \\
\text { Porcentajes }\end{array}$ \\
\hline
\end{tabular}

\section{Fuente: MPPE (2015)}

La sustentación del currículum y la organización que acabamos de mostrar brindan al docente una plataforma para una enseñanza de la Estadística en Educación Media que permita a los estudiantes captar el papel que ésta desempeña en la comprensión de los fenómenos naturales y sociales y para la creación de escenarios de aprendizaje que privilegien el desarrollo de la alfabetización, el razonamiento y el pensamiento estadístico a través de la investigación estadística. A esto se unen algunas herramientas de apoyo que brinda el sistema educativo venezolano como los textos de la Colección Bicentenario que de manera gratuita se entregan a todos los estudiantes, pero a las que no se les saca el debido provecho en las aulas (León y Vicent, 2015)

Precisamente el texto de Matemática de segundo año de Educación Media de esa colección incluye una lección titulada ¿Qué estás bebiendo? (MPPE, 2014, pp. 192-211), donde 
se desarrollan los contenidos de Estadística contemplados en la unidad académica $\mathrm{N}^{\circ} 1$ que se muestra en el cuadro 1 , y que además puede servir de guía para la unidad $\mathrm{N}^{\circ} 5$, siguiendo el esquema de una investigación estadística.: Formulación de preguntas, recolección de datos, análisis de los datos e interpretación de resultados (Wild y Pfanncuch, 1999; Franklin et al, 2005)

Partiendo de una lectura inicial sobre el consumo de bebidas en los seres humanos, donde se abordan tanto los beneficios del consumo de agua y bebidas nutritivas como los riesgos para los adolescentes de ingerir bebidas alcohólicas, se plantea como pregunta de investigación: ¿Qué están bebiendo tú, tus compañeras y compañeros del liceo y las vecinas y vecinos que se encuentran entre 10 y 17 años de edad? A partir de allí se procede a discutir aspectos conceptuales sobre el procesamiento estadístico de la información: recolección, procesamiento, presentación y análisis de datos. A continuación se diseña el plan de recolección de datos y se discute cómo aplicarlo: cuáles datos recolectar, selección de la muestra, métodos e instrumentos de recolección (observación, cuestionario, encuesta). La siguiente fase que se plantea es el análisis de los datos: procesamiento de la información (registro, organización y tabulación de la información), selección y aplicación de métodos gráficos (Gráficos de barra, de sectores y polígonos de frecuencia) y métodos numéricos: medidas de resumen como mediana, mediana, moda, rango. Por último se realiza el análisis de la información a la luz de la pregunta inicial y la comunicación de los resultados (presentación de un ensayo donde destaquen los aspectos positivos del consumo de algunas bebidas y los efectos negativos del consumo de bebidas alcohólicas). Todo esto se va tratando en relación al problema en estudio, con la debida orientación a través de preguntas y sugerencias y promoviendo la participación activa y colaborativa de los estudiantes en las decisiones sobre cada punto. Para cerrar la lección se invita a los estudiantes a plantearse otros temas de interés para la indagación estadística que les permita fortalecer los aprendizajes y habilidades del pensamiento y el razonamiento estadístico logrados en esta lección.

Como se ve, esta lección orienta la enseñanza y el aprendizaje de contenidos fundamentales de la disciplina en el contexto de una investigación, lo que propiciará la creación de significados por el estudiante y redundará en el desarrollo de otros proyectos y en su aplicación en situaciones de su cotidianidad. 
Pero, qué ocurre en la práctica de la enseñanza de la Estadística en Venezuela. En el currículum anterior los contenidos de Estadística y Probabilidad estaban ubicados al final de los programas de Matemática de Educación Media, los cuales seguían una secuencia lineal de obligatorio cumplimiento en las planificaciones docentes. Estos contenidos en general no eran tratados en clases por diversas razones señaladas por los docentes, entre ellas lo extenso de los programas, el reducido tiempo disponible para desarrollarlos, la relevancia de otros temas por sobre los de estas áreas para la prosecución de los estudios a nivel universitario. (Salcedo, 2008).

Cabe entonces preguntarse si con la implantación del nuevo diseño curricular, esta situación se revirtió porque, como lo estipula la fundamentación pedagógica del diseño "el programa lo hace el profesor" (MPPE, 2015, p. 5) siguiendo las orientaciones expresadas en la organización curricular mostrada. Lamentablemente la respuesta a esta interrogante es un rotundo NO. En las aulas de Matemática en Venezuela, la Estadística y la Probabilidad siguen siendo las grandes ausentes, son contados los profesores que las enseñan y éstos en general lo hacen, como ya hemos dicho, poniendo énfasis en la aplicación de fórmula y cálculos (Salcedo, 2008). A pesar de las recomendaciones de trabajar con proyectos interdisciplinarios que permitan posicionar la Estadística como un método de investigación que coadyuva a la comprensión del mundo (Moore, Mc Cabe y Craig, 2009), el docente de Matemática al enseñar Estadística en Educación Media sigue el estilo de enseñanza directa donde el profesor es la autoridad que explica, muestra ejemplos y asigna ejercicios rutinarios, ficticios y de respuesta única; los estudiantes, por su parte, aprenden cómo hacer las cosas y responden a las preguntas que hace el profesor en la forma que éste espera que lo hagan (Ponte, 2011)

Son pocos los liceos donde se aplica el método de proyectos como vía para una enseñanza interdisciplinar y contextualizada (Azócar, 2013) y donde lo hacen parece no haber claridad sobre como incorporar en ellos los contenidos del área de Matemática, como veremos a continuación.

En una investigación reciente llevada a cabo en un liceo del Estado Monagas, titulada "Proyectos de aprendizaje y la enseñanza de la Matemática" (Guanipa, 2019), se reportan algunas experiencias al respecto. En este liceo, en cada período académico cada sección planifica un proyecto de aprendizaje (P.A.) dentro del cual se abordan los referentes teóricoprácticos de las diferentes áreas, sin embargo para los profesores de Matemática “la 
planificación por proyectos es un proceso no del todo comprendido", "la contextualización un escollo difícil de superar"; "el tejido temático es un embrollo interdisciplinar" y "la matemática no encaja en la planificación conjunta de los proyectos", según los testimonios de los sujetos informantes de esa investigación (Op, cit, p. 82).

En el primer lapso del año 2018 una de las secciones de segundo año escogió un P.A. titulado "Errores del pasado, consecuencias del presente y soluciones para el futuro". El profesor de Matemática decidió desarrollar la primera unidad del programa, organizada en el currículum como se muestra en el cuadro 2 .

Cuadro 2: Unidad de aprendizaje $\mathrm{N}^{\circ} 1$ del programa de Matemática de 2do año de Educación Media

\begin{tabular}{|c|c|c|}
\hline $\mathbf{U A}$ & Tema generador-Tejido temático & Referentes teórico-prácticos \\
\hline 1 & $\begin{array}{l}\text { Dinámica y distribución de las poblaciones humanas } \\
\text { La población de Venezuela a lo largo de los siglos XX y XXI: } \\
\text { total de habitantes, población por sexo, grupo de edad, entidad } \\
\text { federal, densidad de población, media y mediana de edad. } \\
\text { Comparación con población mundial, con la población de otros } \\
\text { países de América latina y del mundo- }\end{array}$ & $\begin{array}{l}\text { Tablas de doble entrada } \\
\text { Representaciones gráficas de } \\
\text { proporciones, histograma, } \\
\text { gráficas de línea. } \\
\text { Medidas de tendencia central: } \\
\text { medias y medianas. } \\
\text { Estimaciones } \\
\text { Densidad, población, tasa } \\
\text { Unidades de medida } \\
\text { Porcentajes } \\
\text { Fracciones como proporciones }\end{array}$ \\
\hline
\end{tabular}

Fuente: MPPE (2015)

A tal efecto, utilizó un esquema de planificación (cuadro 3) que incluye, junto con el tema generador y los referentes teórico-prácticos, los siguientes elementos:

1) Conceptualización: construcción teórica a partir del tema específico que se estudia

2) Generalización: "Los temas generadores hacen planteamientos que permiten las generalizaciones tanto en el espacio como en el tiempo, de lo local a lo global, de lo particular a lo general"'.

3) Sistematización: invita a los docentes que "favorezca espacios para que los y las estudiantes sistematicen lo aprendido y los procesos realizados para comprender lo estudiado, tomando conciencia de sus propios procesos (metacognición)”. (MPPE, 2015, p. 92) 
Cuadro 3: Conceptualización, generalización y sistematización en la planificación de la UA $\mathrm{N}^{\circ} 1$ presentada por el profesor de Matemática

\begin{tabular}{|c|c|c|}
\hline Conceptualización & Generalización & Sistematización \\
\hline $\begin{array}{l}\text { Ejercitación en la } \\
\text { elaboración de } \\
\text { gráficas. Calculo de } \\
\text { porcentajes, } \\
\text { familiarización con } \\
\text { la conversión de } \\
\text { unidades, } \\
\text { transformaciones } \\
\text { en el plano, } \\
\text { congruencia de } \\
\text { figuras planas. } \\
\text { Graficas de } \\
\text { dispersión. }\end{array}$ & $\begin{array}{l}\text { La población de Venezuela a } \\
\text { lo largo de los siglos XX y XXI: } \\
\text { en relación a los alimentos que } \\
\text { ingiere la población por sexo, } \\
\text { grupos de edad y entidad federal, } \\
\text { densidad de población, medias y } \\
\text { medianas de edad. } \\
\text { Comparación con población } \\
\text { mundial, con la población de otros } \\
\text { países de América Latina y el } \\
\text { mundo. }\end{array}$ & 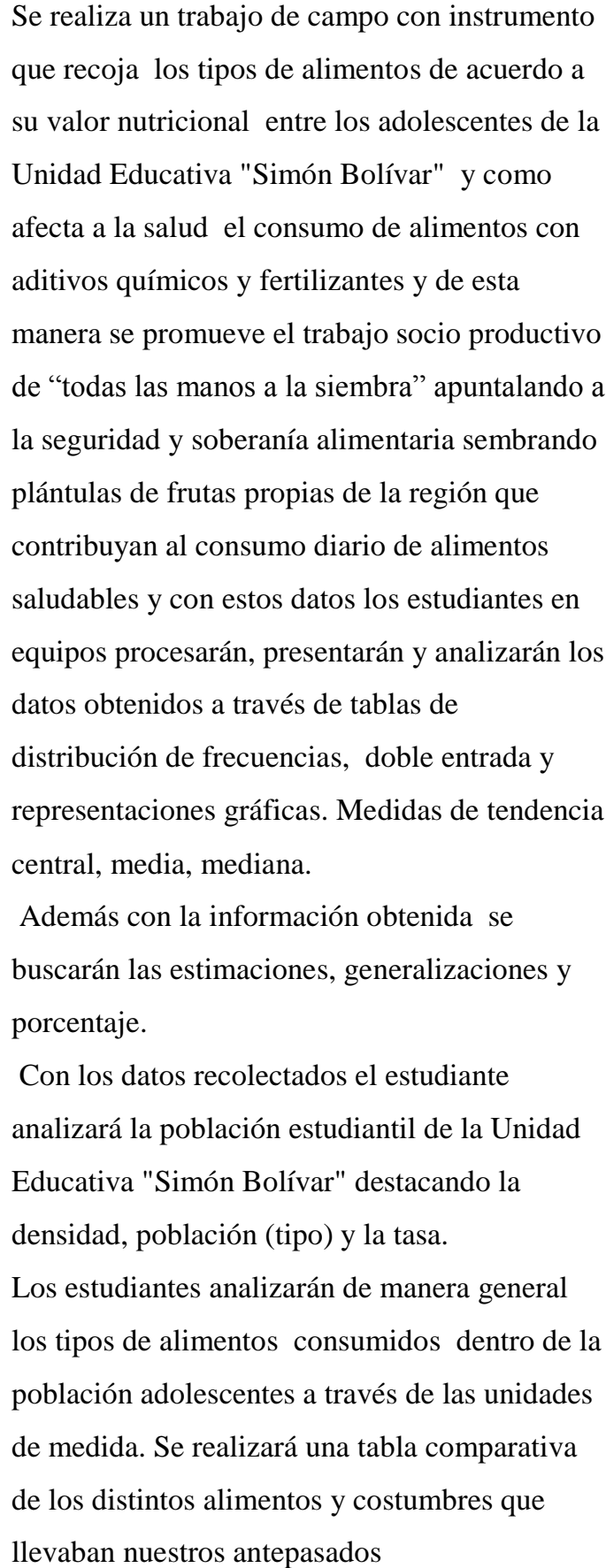 \\
\hline
\end{tabular}

Fuente: Guanipa (2019)

$\mathrm{Al}$ analizar esta planificación, lo primero que se observa es una falta de correspondencia entre el tema generador y las indicaciones para la generalización y la sistematización. El tema generador es "Dinámica y distribución de las poblaciones humanas", a partir del cual se 
deberían estudiar los referentes teóricos-prácticos del área de Estadística contextualizados a un tejido temático que entrelaza con el campo de la Geografía, a través de la Demografía. La Demografía estudia el estado y la dinámica de la población; refiriéndose el estado a su conformación por sexo, edad y otros indicadores y a su tamaño y distribución geográfica; y la dinámica a los cambios en la estructura poblacional según la natalidad, la mortalidad y la migración (Paladino, 2010). En consecuencia, la generalización y la sistematización deben estar en concordancia con estos dos aspectos conceptuales que derivan de dicho tema generador; sin embargo, éstas se dirigen a estudiar cuestiones relacionadas con la nutrición de los estudiantes del liceo y su incidencia en su salud, lo que estaría más acorde con el tema generador de la UA $\mathrm{N}^{\circ} 5$ de $1^{\circ}$ año mostrada en el cuadro 1 .

El tema generador "Dinámica y distribución de las poblaciones humanas" brinda un escenario de aprendizaje muy rico para el desarrollo de conceptos relacionados con el análisis de datos a través de la indagación; por ejemplo, se puede estudiar la distribución de la población por edad, sexo, ubicación geográfica, entre otros indicadores; a través de su organización en tablas de frecuencia con valores absolutos, porcentuales y acumulativos se puede tener una mejor comprensión de tales distribuciones; representaciones gráficas usuales como histogramas y gráficos de líneas posibilitan una mejor visualización del comportamiento de las variables de interés y detectar patrones y tendencias que permitan hacer inferencias informales; diagramas de caja y bigote también mostrarán una visión clara de dichas distribuciones en cuanto a centro y variabilidad y facilitarán las comparaciones entre grupos. Obviamente este análisis requerirá el cálculo de valores de resumen como medias, medianas, percentiles, además de indicadores propios de la demografía como índices de natalidad, mortalidad, entre otros. Todas estas tareas pueden realizarse con el uso de la tecnología.

Igualmente, como lo pide el tejido temático, se pueden hacer comparaciones con otros países con respecto a variables como la edad seguidas de una discusión, por ejemplo, sobre los retos de cada país en materia de educación y salud para la atención de las personas según los grupos etarios predominantes. En el libro "Enfoque de las Matemáticas para la Educación Media Superior. Razonamiento y construcción de significados. Estadística y Probabilidad", Shaughnessy, Chance y Kranendock (2017) presentan una tarea titulada "Datos de países: Revisión de datos censales", en la que brindan a los docentes pautas de actuación didáctica para llevar a los estudiantes a desarrollar habilidades y hábitos mentales relacionados al 
razonamiento estadístico, como descubrir y describir patrones, buscar estructuras ocultas de los datos y generar deducciones y conjeturas. (Ob, cit, 2017),

Hay que resaltar que en el proyecto que venimos analizando, el punto álgido de la investigación estadística, referido a la generación de los datos, no estará aquí presente pues se ha de usar datos sobre la población venezolana durante los siglos XX y XXI disponibles en la página WEB del Instituto Nacional de Estadística (www.ine.gov.ve ). Se ha de trabajar con datos censales, lo que facilitará a los estudiantes establecer diferencias entre poblaciones y muestras y las diferentes formas de pensar sobre estos tipos de datos: censales y muestrales. Por supuesto, habrá que determinar cuál y cuánta de la vasta gama de información disponible se requiere para responder las preguntas o realizar las tareas que se hayan planteado. Estas preguntas o tareas pueden surgir del consenso entre los estudiantes orientados por el profesor o, simplemente, propuestas por éste. Igualmente, la organización y análisis de los datos requeridos en cada fase de las tareas deben ser monitoreadas por el docente quien propiciará la reflexión, el razonamiento y la construcción de significados en los estudiantes, sin perder de vista la vinculación al contexto donde se sitúa el estudio.

Tomando en cuenta que la unidad de aprendizaje que se analiza se desarrolla en el marco del proyecto "Errores del pasado, consecuencias del presente y soluciones para el futuro", el profesor deberá orientar a los estudiantes, a través de un ciclo de preguntas, para que logren extraer "errores" que hayan ocurrido en la dinámica y la distribución de la población venezolana en el período en estudio, cómo estos afectan nuestra vida en la actualidad y las medidas que podrían tomarse para revertir esas situaciones indeseadas. No será suficiente con que los estudiantes sean capaces de elegir convenientemente gráficos apropiados para una situación particular, que sepan construirlos e interpretarlos y hacer conjeturas e inferencias, sino que esto se haga a la luz del momento socio-histórico en que ocurrieron los hechos, sus causas y consecuencias.

Me permito, haciendo quizás otra digresión, referirme a uno que a mi parecer ha sido de los más grandes y graves errores que este estudio debería develar a los estudiantes: el abandono del campo con las migraciones internas desde este hacia las ciudades ocurridas a partir de la segunda década del siglo pasado cuando se inició la actividad petrolera en nuestro país. De ahí en adelante comenzó a desaparecer la Venezuela rural y la actividad agrícola entró en franco declive. Han sido muchos los escritores y estudiosos de la realidad venezolana, entre ellos 
Arturo Uslar Pietri, Rodolfo Quintero, Julián Padrón y Miguel Otero Silva, quienes en su momento advirtieron sobre las consecuencias nefastas de esta política para el país, pero éstas cayeron en saco roto ante el flujo de las divisas del petróleo que se despilfarraron a granel, con las consecuencias que ahora están a la vista.

Los estudiantes deben conocer estos hechos a través del análisis de la información estadística, discutir sobre ellos y formarse su propia opinión al respecto, al poner en acción su pensamiento estadístico y, desde una postura crítica, comprometerse con las soluciones para el futuro, con las transformaciones que deben producirse para sobreponernos a los daños infligidos a la nación y sus ciudadanos. Huelga decir que este es un tema que en Venezuela genera mucha incertidumbre.

Esto pasa por un conocimiento de la historia política, económica y social del país. Al inscribirse esta temática en el proyecto de aula de una sección de segundo año de bachillerato y siendo Petróleo y Energía uno de los "temas indispensables" que debe permear todas las áreas del currículum (MPPE, 2015), el docente de Matemática debería trabajar mancomunadamente con los profesores de otras áreas como Lengua y Sociales, para darle un enfoque interdisciplinar al asunto. Por ejemplo sería deseable que el profesor de Lengua promoviera la lectura de las novelas “Oficina $N^{\circ} 1$ ” (Otero Silva, 1961) y “Clamor campesino" (Padrón, 1945) y que el profesor de Sociales hiciera lo propio con las ideas sobre "La siembra del petróleo" de Arturo Uslar Pietri (s.f.) y la obra "La Cultura del Petróleo" (Quintero, 1972), entro otros aportes. Estas obras recrean la situación planteada.

Como vemos, son muchas las ideas que circundan este proyecto; son amplias las posibilidades para llevar a cabo una práctica de la estadística contextualizada que conduzca al desarrollo del pensamiento y el razonamiento estadístico, pero éstas solo pueden materializarse con una buena formación del docente que enseña estadística en Educación Media. En caso contrario, aun cuando esta enseñanza se de en el marco de un proyecto de aprendizaje, tal como lo muestran los estudios sobre el desarrollo profesional de los profesores, es muy probable que el docente se centre en las características procesales de la investigación estadística o se atasque en las fases de formulación del problema y la recopilación de datos (Ponte y Noll, 2018). Esto es lo que ha ocurrido en la situación que se viene analizando donde no hubo una buena formulación de los propósitos de la investigación y en realidad, como muestra la planificación 
semanal, se hizo énfasis en aspecto procedimentales, tanto en el desarrollo de la clase como en la evaluación (ver Figura 1).

Figura 1: Planificación semanal del profesor de Matemática

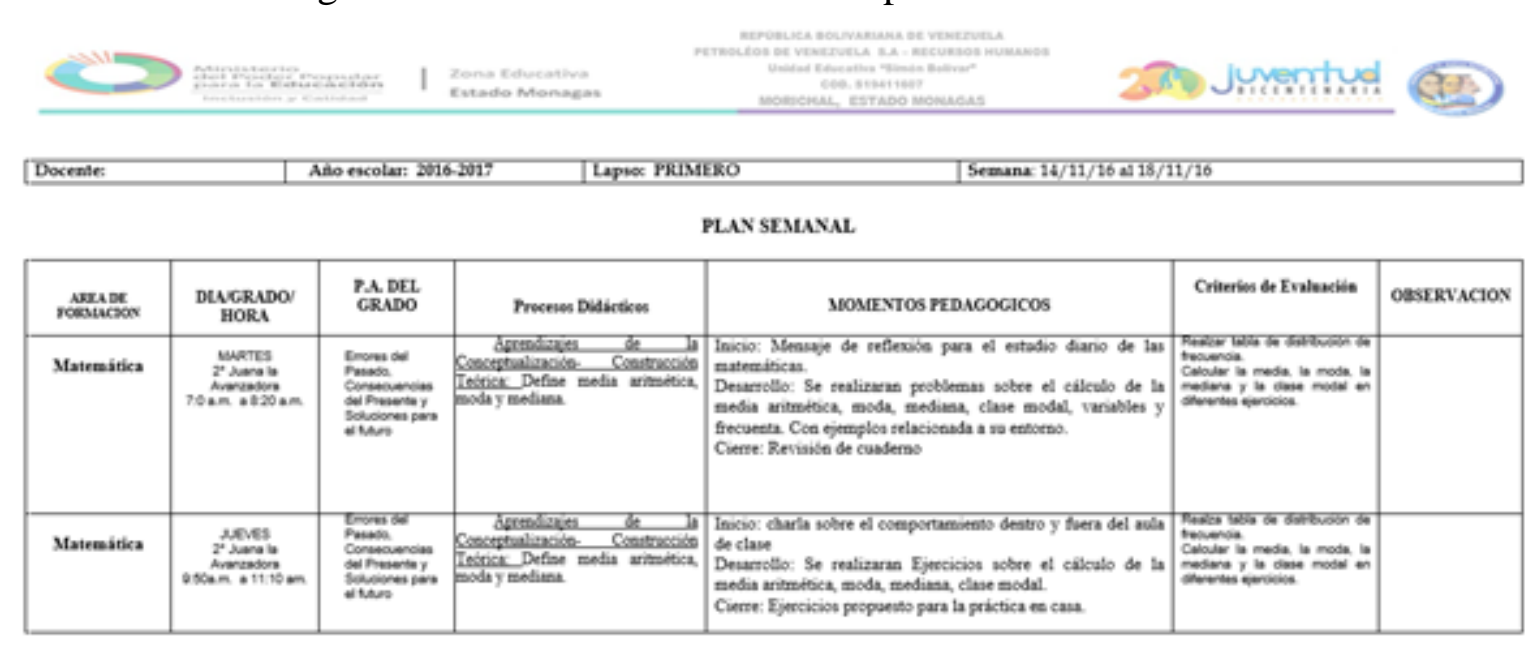

Fuente: Guanipa (2019)

Para cerrar este apartado, acotamos que las actividades de generalización y sistematización planificadas por el docente bien se adaptan a la $\mathrm{UA} \mathrm{N}^{\circ} 5$ del programa (Cuadro 1), cuyo tema generador es Alimentación y nutrición. Este es un tema muy propicio para una investigación estadística que permita profundizar la comprensión de los conceptos incluidos en los referentes teórico-prácticos: Tablas, gráficas, media aritmética, razones, proporciones, índices y porcentajes. Esta investigación además brindaría la oportunidad de abarcar la fase de planificación de la recolección de datos y su ejecución, coadyuvando al desarrollo de uno de los tipos de pensamiento del modelo de Wild y Pfannkuch (1999), Reconocimiento de la necesidad de los datos, algunos de cuyos indicadores son: reconocer la necesidad y utilidad de los datos; caracterizar correctamente la muestra; recolectar apropiadamente los datos (Pimienta, 2006, citado por Insunza, 2017).

En el libro de Matemática de primer año de la Colección Bicentenario, en la lección titulada "Programa de Alimentación Escolar", (MPPE, 2013, pp. 206-217), se desarrolla el tema "Datos estadísticos y medidas de tendencia central" a través de una investigación estadística que busca responder preguntas sobre las preferencias alimenticias de los estudiantes y la calidad de la comida que sirven en el comedor escolar. Esta es una buena guía para el docente interesado en involucrar a sus estudiantes en la ejecución de proyectos e investigaciones estadísticas, 
entonces nos preguntamos por qué no lo hace y la respuesta parece estar en una deficiente formación conceptual, la inseguridad para conducir exitosamente a sus estudiantes y una actitud poco favorable hacia la estadística y su enseñanza (León, 2019).

\section{Para cerrar: lo que requiere atención primordial}

Después de haber abordado algunas circunstancias que limitan la formación de un ciudadano estadísticamente educado en Venezuela, quiero cerrar este escrito refiriéndome a dos cuestiones que hay que atender en la Educación Estadística en Venezuela.

La primera de ellas tiene que ver con la preparación de los docentes que enseñan Estadística en Educación Media. Estos profesores no comprenden claramente las diferencias entre la Matemática y la Estadística y por esa razón enseñan los contenidos estadísticos como lo hacen con los contenidos matemáticos, sin tomar en cuenta las particularidades del pensamiento y el razonamiento estadísticos. Estepa y Gea (2010, p. 23) afirman que "no cabe un desarrollo espontáneo en el razonamiento estadístico y probabilístico del estudiante sin un adecuado proceso de instrucción”, por lo que Pfannkuch (2008) sugiere formar al docente para que "tome conciencia del poder que posee en cuanto a la posibilidad de desarrollar la capacidad de razonamiento de sus estudiantes, con la consecuente repercusión en la comprensión conceptual de los temas tratados" (p. 24).

En el Proyecto Gaise (Franklin et al, 2005) se asegura que la Estadística es para muchos docentes un área relativamente nueva, sobre la cual "no han tenido oportunidad de desarrollar un conocimiento sólido de principios y conceptos subyacentes a las prácticas de análisis de datos que ahora están llamados a enseñar" (p. 5). En el caso de Venezuela, como ya hemos dicho, la mayoría de los docentes que actualmente enseñan Estadística en Educación Media son profesores de Matemática egresados principalmente de la Universidad pedagógica Experimental Libertador (UPEL). Estos han sido formados a través de un currículo (que fue transformado en 2017) integrado por cuatro componentes separados entre sí: formación general, formación especializada, formación pedagógica, y formación para la práctica profesional. Ese diseño curricular contempla en el componente de formación pedagógica un curso de Estadística Educativa en el que solo se abarcan temas de Estadística Descriptiva, y en el componente de formación especializada uno de Probabilidad y Estadística Inferencial, con formalidad matemática pero tan extenso en contenido que solo se llegan a cubrir los de Probabilidad mas 
no los de Estadística Inferencial. No hay cursos de didáctica de la Probabilidad y Estadística. (León, Beyer, Serres e Iglesias, 2013).

Tal formación no les ha permitido lograr ni el conocimiento conceptual ni el conocimiento pedagógico del contenido para la enseñanza de la Estadística en Educación Media. Ellos no han tenido una experiencia formativa que contemple los temas y habilidades claves a desarrollar a través de la Educación Estadística en la actualidad: comprensión de los conceptos y las ideas fundamentales de la Estadística; habilidad para explorar y aprender de los datos; desarrollo de la argumentación estadística; uso de una evaluación formativa y comprensión de las formas de pensar y razonar de la Estadística (Pfannnkuch y Ben-Zevi, 2008). Tampoco han tenido oportunidad de una práctica estadística con el uso de tecnología. Hay un desconocimiento casi total de toda la gama de software disponible a tal efecto.

Sería deseable, entonces, un cambio de concepción en la formación del docente que ha de enseñar Estadística hacia un currículum que integre el conocimiento conceptual de la disciplina y el conocimiento profesional para enseñarlo con miras a desarrollar una cultura estadística en sus estudiantes Este currículum debe contemplar el desarrollo de las grandes ideas de la Estadística, del pensamiento y el razonamiento estadístico de los propios profesores y el uso de la tecnología. (Ben-Zevi y Garfield, 2004; Garfield y Ben-Zevi, 2008; Godino, Ortiz, Roa y Wilhelm, 2011, Ponte y Noll, 2018; Makar y Fielding-Wells, 2011; Burgess, 2008).

En la formación del futuro profesor de Matemática de la UPEL hacia una enseñanza de la Estadística a través de la investigación se sugiere el modelo de Burgess, resumido en el cuadro 5, el cual abarca por un lado las categorías del conocimiento del profesor dentro de la Teoría Matemática para la Enseñanza (Ball, Thames y Phelps, 2005) - conocimiento del contenido: conocimiento común y conocimiento especializado; conocimiento pedagógico del contenido: conocimiento del contenido y los estudiantes y conocimiento del contenido y la enseñanza -, derivadas de las ideas iniciales de Shulman (1986); y por el otro, las cuatro dimensiones del pensamiento estadístico en la investigación empírica identificadas por Wild y Pfannkuch (1999). Este modelo debe ponerse en acción en ambientes educativos computarizados. 
Cuadro 5: Marco para examinar la preparación el docente para la enseñanza de la Estadística a través de la investigación.

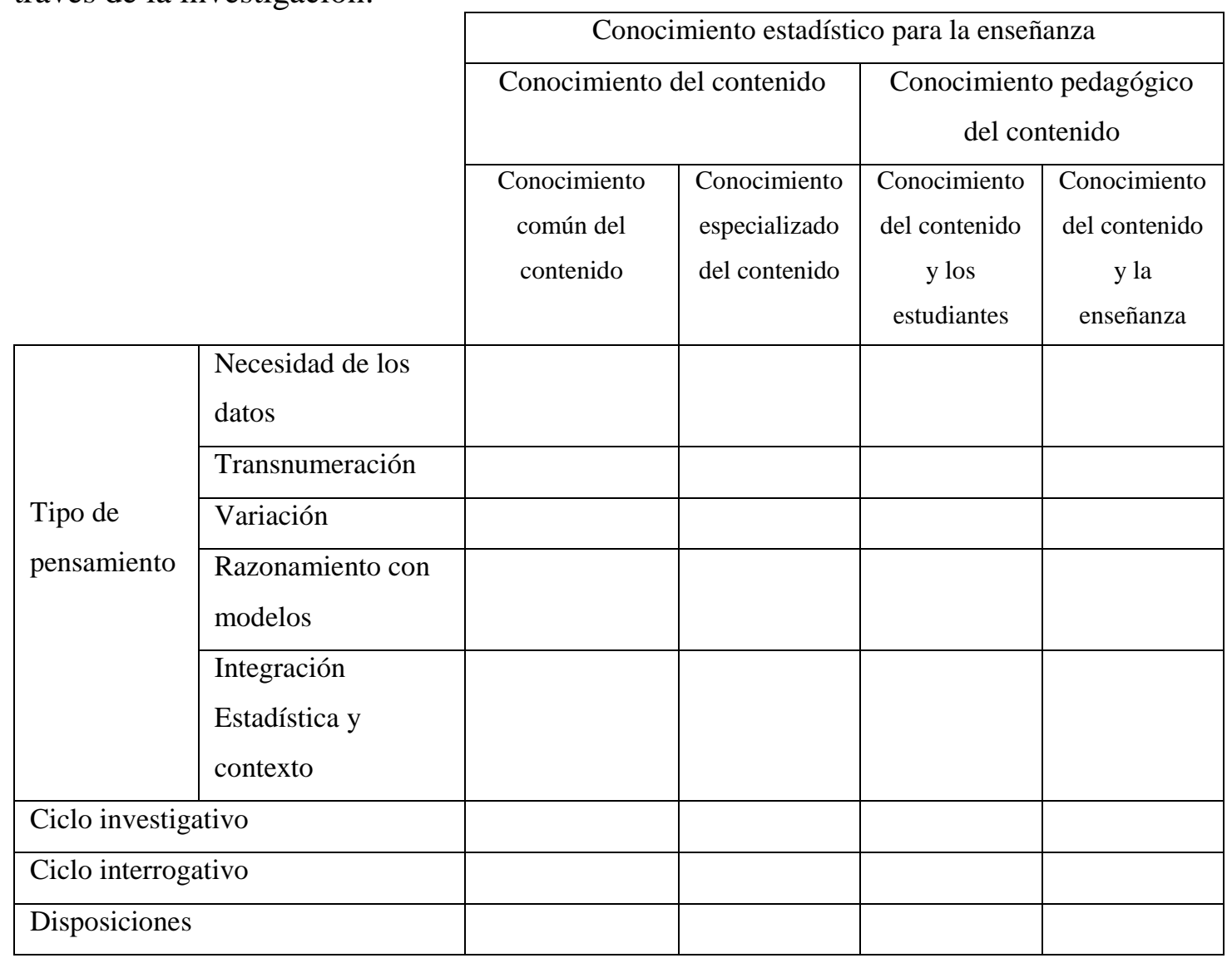

Fuente: Adaptado de Burgess (2008)

La segunda cuestión que amerita atención para lograr trasformaciones en la enseñanza y el aprendizaje de la Estadística se refiere a la afectividad. El conocimiento conceptual y el conocimiento pedagógico del contenido son necesarios, pero no suficientes; se requiere del acompañamiento de un componente afectivo que abarca actitudes, creencias, motivaciones y emociones, pues éste incide directamente en el aprendizaje estudiantil (Batanero, 2011). Una actitud no favorable, una falsa creencia sobre la Estadística y su aprendizaje como por ejemplo el pensar que el conocimiento estadístico carece de significado más allá del aula y de aplicabilidad a la vida real, generará emociones negativas que bloquearán el aprendizaje y el razonamiento estadístico. En este sentido, el docente deberá trabajar primero en sí mismo sobre el logro de una afectividad personal positiva hacia la Estadística y su enseñanza para luego proyectarla hacia sus estudiantes. 
Trabajando sobre estos dos puntos de atención, seguramente los resultados serán más halagadores.

\section{Referencias}

Azcárate, P. y Cardeñoso, J.M. (2011). La enseñanza de la Estadística a través de escenarios: implicaciones en el desarrollo profesional. Bolema, Rio Claro (SP), 24 (40), 789-810.

Azocar, K. (2013, Julio 25). Análisis de problemáticas sociales locales con el uso del programa informático CALC en el estudio de la estadística descriptiva. [Trabajo de grado de Maestría]. UPEL-IPM, Maturín. Universidad Pedagógica Experimental LibertadorInstituto Pedagógico de Maturín. Venezuela.

Batanero, C. (2000). ¿Hacia dónde va la educación estadística? Blaix, 15, 2-13.

Batanero, C. (2002). Presente y futuro de la Educación Estadística. [En línea]. Recuperado Enero 25, 2020 de www.researchgate.net > publication > 256296106_Presente_y_Futuro_d...

Batanero, C. (2004). Los retos de la cultura estadística. Yupana, 1(1), 27-37.

Batanero, C. (2011). Joint ICMI/IASE Study: Teaching Statistics in School Mathematics. Challenges for Teaching and Teacher Education. [En línea]. Recuperado Enero 25, 2020 de www.ugr.es > CIAEM_ICMIStudy_Batanero.

Batanero, C. y Díaz, C. (2011). Estadística con Proyectos. [En línea]. Recuperado Enero 25, $2020 \mathrm{de}$ http://www.ugr.es/ batanero/pages/ARTICULOS/Libroproyectos.pdf

Batanero, C. y Godino, J. (2005). Perspectivas de la Educación Estadística como área de investigación. En R. Luengo (Ed.) Líneas de investigación en didáctica de las matemáticas (pp. 203-226). Badajoz, Universidad de Extremadura.

Ben-Zevi, D. y Garfield, J. (2004). Statistical literacy, reasoning, and thinking: goals, definitions, and challenges. En D. Ben-Zevi y J. Garfield (Eds.) The challenge of developing statistical literacy, reasoning and thinking (pp. 3-15). Dordrecht, The Netherlands: Kluwer

Ben-Zvi, D., y Makar, K. (2016). The teaching and learning of Statistics. International perspectives. Switzerland: Springer International Publishing.

Ball, D., Thames, M. y Phelps, G. (2005). Articulating domains of mathematical knowledge for teaching. [En línea] Recuperado Enero 28, 2020 de www-personal.umich.edu/ dball/

Burgess, T. (2008). Teacher knowledge of and for Statistical investigation. En C. Batanero, G. Burrill, C. Reading, C., y A. Rossman, (Eds.), Joint ICMI/IASE Study: Teaching Statistics in school Mathematics. Challenges for teaching and teacher education. Proceedings of the ICMI Study 18 and 2008 IASE Round Table Conference. (pp. 259-270). Monterrey: ICME/IASE.

Burril, G. y Bielher, R. (2011). Fundamental statistical ideas in the school curriculum and in training teachers. En C. Batanero, G. Burril, C. Reading (Eds.), Teaching Statistics in school Mathematics - Challenges for teaching and teacher education (pp. 57-69). New York: Springer. 
Chance, B. L. (2002). Components of statistical thinking and implications for instruction and assessment. Journal of Statistics Education [En línea], 10(3). Recuperado Enero 15, 2020 de http://www.amstat.org/publications/jse/

delMas, R. (2002). Statistical literacy, reasoning, and learning: A commentary. Journal of Statistics Education [En línea], 10(3). Recuperado Enero 15, 2020, de http://www.amstat.org/publications/jse/

delMas, R. (2004). A comparison of mathematical and statistical reasoning. En D. Ben-Zevi y J.Garfield (Eds.), The Challenge of Developing Statistical Literacy, Reasoning and Thinking (pp. 79-95). Dordrecht, The Netherlands: Kluwer Academic Press.

Díaz, D., Aguayo, C. y Cortez, C. (2014). Enseñanza de la estadística mediante proyectos y su relación con las teorías de aprendizaje. Revista Premisa, 16(62), 16-23.

Díaz, D. y Sánchez, J. (2011). Aplicando Estadística en problemas actuales. Revista Premisa, $51,37-48$.

Estepa y Gea (2010). Conocimiento para la enseñanza de la asociación estadística. En J. Ortiz (Ed.) Investigaciones actuales en Educación Estadística y formación de profesores. (pp. 23-40). Departamento de Didáctica de la Matemática. Universidad de Granada.

Estrella, S. (2017). Enseñar Estadística para alfabetizar estadísticamente y desarrollar el razonamiento estadístico. En A. Salcedo, Alternativas pedagógicas para la Educación Matemática del Siglo XXI (pp. 173-194). Caracas: Centro de Investigaciones Educativas, Escuela de Educación. Universidad Central de Venezuela.

Franklin, C., Kader, G., Mewborn, D., Moreno, J., Peck, R., Perry, M. y Scheaffer, R. (2005). Lineamientos para la evaluación y enseñanza en Educación Estadística. Proyecto GAISE. Un marco para el currículum de Pre-K-12. Alexandría, VA: American Statistic Association.

Gal, I (2002). Adult's statistical literacy. Meanings, components, responsibilities. International Statistical Review, 70(1), 1-25.

Garfield, J. (2002). The challenge of developing statistical reasoning. Journal of Statistics Education [En línea], 10(3). Recuperado Enero 15, 2020, de http://www.amstat.org/publications/jse/

Garfield, J. y Ben-Zvi, D. (2008). Developing students' statistical reasoning: Connecting research and teaching practice. Dordrecht, The Netherlands: Springer

Gattuso, L. y Ottaviani, M. (2008). Complementing mathematics and statistical thinking in school mathematics. En C. Batanero, G. Burrill, C. Reading, C., y A. Rossman, (Eds.). Joint ICMI/IASE Study: Teaching Statistics in school Mathematics. Challenges for teaching and teacher education. Proceedings of the ICMI Study 18 and 2008 IASE Round Table Conference, (pp. 121-132). Monterrey: ICMI/IASE.

Godino, J., Ortiz, J. Roa, R. y Wilhelm, R. (2011). Models for Statistical Pedagogical Knowledge. En C. Batanero, G. Burrill, y C. Reading (Eds.), Teaching Statistics in School Mathematics Challenges for Teaching and Teacher Education. A Joint ICMI/IASE Study: The 18th ICMI Study (pp. 271-282). New York: Springer 
Guanipa, Y. (2019, Agosto 23). Proyectos de aprendizaje y la enseñanza de la Matemática. [Trabajo de grado de Maestría]. UPEL-IPM, Maturín.

Insunza, S. (2014). Razonamiento estadístico en estudiantes universitarios sobre el análisis de datos en ambiente computacional. Bolema, Rio Claro (SP), 28(50),1262-1286.

Insunza, S. (2017). Potencial de los proyectos para desarrollar motivación, competencias de razonamiento y pensamiento estadístico. Revista Actualidades investigativas en educación, 17(3), 1-30.

León, N. (2019). Textos escolares desde una visión crítica de la matemática. Cuadernos de Investigación y Formación en Educación Matemática., año 14, № 18, 90-104.

León, N., Beyer, W., Serres, Y. e Iglesias, M. (2013). Informe sobre la formación inicial y continua de profesores de Matemática. Venezuela. Cuadernos de Investigación y Formación en Educación Matemática, Año 8, Número Especial, 89-129.

León, N. y Vicent, R. (2015). Valoración de los textos de matemática de educación media de la colección bicentenario desde la perspectiva de docentes y estudiantes de la especialidad. Informe de Investigación presentado a la Subdirección e Investigación y Postgrado de la UPEL-IPM. Maturín: Autor.

MacGillivray, H., y Pereira-Mendoza, L. (2011). Teaching Statistical Thinking Through Investigative Projects. En C. Batanero, G. Burrill, y C. Reading (Eds.), Teaching Statistics in School Mathematics Challenges for Teaching and Teacher Education. A Joint ICMI/IASE Study: The 18th ICMI Study (pp. 109-120). New York: Springer.

Makar, K., y Fielding-Wells, J. (2011). Teaching teachers to teach statistical investigations. En C. Batanero, G. Burrill y C. Reading (Eds.), Teaching statistics in school mathematics Challenges for teaching and teacher education: a joint ICMI/IASE study: the 18th ICMI study (pp. 347-358). Dordrecht: Springer.

Ministerio del Poder Popular para la Educación - MPPE (2013). Matemática para la vida. Primer año. Caracas: Autor.

Ministerio del Poder Popular para la Educación - MPPE (2014). Conciencia matemática. Caracas: Autor.

Ministerio del Poder Popular para la Educación - MPPE (2015). Proceso de cambio curricular en Educación Media. Documento general de sistematización de las propuestas pedagógicas y curriculares surgidas en el debate y discusión. Caracas: Autor.

Moore, D., McCabe, G. y Craig, B. (2009). Introduction to the practice of Statistics. Sixth edition. New York: W.H. Freeman and Company.

Moore, D. y Cobb, G. (1997). Mathematics, Statistics, and Teaching. American Mathematical Monthly, 104, 801-823.

National Council of Teachers of Mathematics (2000). Principles and standards for school mathematics. Reston, VA: Author. [En línea]. Recuperado Enero 25, 2020 de http://standards.nctm.org/

Otero Silva, M. (1961). Oficina $N^{\circ}$ 1. Caracas: Editorial Losada.

Padrón, J. (1945). Clamor campesino. Caracas: Editorial Élite. 
Palladino, A. (2010). Introducción a la demografía. [En línea] Recuperado Enero 25, 2020 de med.unne.edu.ar $>$ sitio $>$ imagenes $>$ ckfinder $>$ files $>$ files $>$ aps $>$ I...

Pfannkuch, M. (2008). Training teachers to develop statistical thinking. En línea. Recuperado de www.stat.auckland.ac.nz $>\sim$ iase > T4P2 Pfannkuch

Pfannkuch, M. (2011). The role of context in developingInformal statistical inferential reasoning: A classroom study. Mathematical Thinking and Learning, 13(1-2), 27-46.

Pfannkuch, M. y Ben-Zevi, D. (2008). Developing teachers's statistical thinking. En C. Batanero, G. Burrill, C. Reading, C., y A. Rossman, (Eds.). Joint ICMI/IASE Study: Teaching Statistics in school Mathematics. Challenges for teaching and teacher education. Proceedings of the ICMI Study 18 and 2008 IASE Round Table Conference, (pp. 323-334). Monterrey: ICMI/IASE.

Pfanncuch, M. y Wild, C, (2004). Towards an understanding of statistical thinking. En D. Ben-Zevi y J. Garfield (Eds.) The challenge of developing statistical literacy, reasoning and thinking (pp.17-46). Dordrecht, The Netherlands: Kluwer

Ponte, J. (2011). Preparing teachers to meet the challenges of Statistics Education. In C. Batanero, G. Burrill y C. Reading (Eds) Teaching statistics in school mathematics Challenges for teaching and teacher education: A Joint ICMI/IASE Study. New York, NY: Springer

Ponte, J. y Noll, J. (2018). Building Capacity in Statistics Teacher. En D. Ben-Zv, K. Makar y J. Garfield. (Eds.), International Handbook of Research in Statistics Education. (pp. 433455). Springer International Publishing.

Quintero, R. (1972). La cultura del petróleo. México: Siglo Veintiuno Editores.

Scheaffer. R. (2006). Statistics and mathematics: On making a happy marriage. En G. Burrill (Ed.) NCTM 2006 Yearbook: Thinking and reasoning with data and chance (pp. 309-321). Reston, VA: NCTM.

Salcedo, A. (2008). Educación Estadística en Venezuela. El caso de la Educación Básica y Media, ¿Formando una cultura estadística?. Ciencias económicas. Revista de la Facultad de Ciencias Económicas. Universidad Nacional del Litoral, Argentina. 6.02. $47-65$

Sánchez, E. y Hoyos, V. (2013). La Estadística y la propuesta de un currículo por competencias. En A. Salcedo (Ed.) La Educación Estadística en América Latina. Tendencias y perspectivas. (pp. 211 - 227). Programa de Cooperación Interfacultades. Universidad Central de Venezuela,

Shaughnessy, J.M (2019). Recommendations about the Big Ideas in Statistics Education: A retrospective from curriculum and research. Cuadernos de Investigación y Formación en Educación Matemática, $\mathrm{N}^{\circ}$ 18, 44-58.

Shaughnessy, J.M., Chance, B., y Kranendonk, H. (2017). Enfoque de las Matemáticas para la Educación Media Superior. Razonamiento y construcción de significados. Probabilidad y Estadística. NCTM. Reston, VA

Shulman, L. (1986). Those who understand: Knowledge growth in teaching. Educational Researcher, 15(2), 4-14. 
Uslar Pietri, A. (s.f.). La siembra del petróleo. Recuperado de ance.msinfo.info > biblo > texto $>$ libros > BC.1991.T.III.b.2.pdf.

Utts, J. (2003). What educated citizens should know about Statistics and Probability? The American Statistician, 57(2), 74-79.

Wallman, K. (1993). Enhancing Statistical Literacy: enriching our society, Journal of the American Statistical Association, 88(421), 1-8.

Watson, J. (1997). Assessing statistical literacy through the use of media surveys. En I. Gal y J. B. Garfield (Eds.) The assessment challenge in statistics education (pp. 107-121). Amsterdam: IOS Press.

Wild, C. y Pfannkuch, M. (1999). Statistical thinking in empirical enquiry (with discussion). International Statistical Review, 67(3), 223-265.

Wild, C., Utts, J. y Horton, N. (2018). Why Statistics?. En D. Ben-Zvi, K. Makar and J. Garfield (Eds.) International Handbook of Research in Statistics Education, (pp. 5-36). Springer International Publishing. 